\title{
ON COMMUTATIVITY THEOREMS FOR RINGS
}

\author{
H.A.S. ABUJABAL and M.S. KHAN \\ Department of Mathematics \\ Faculty of Science \\ King Abdul Aziz University \\ P.0. BOX 9028, Jeddah - 21413 \\ Saudi Arabia
}

(Received February 2, 1989)

ABSTRACT. Let $R$ be an associative ring with unity. It is proved that if $R$ satisfies the polynomial identity $\left[x^{n} y-y^{m} x^{n}, x\right]=0(m>1, n>1)$, then $R$ is commutative. Two or more related results are also obtained.

KEY WORDS AND PHRASES. Commutative rings, torsion free rings, center of a ring, commutator ideal.

1980 AMS SUBJECT CLASSIFICATION CODES. 16A20, 116 A70.

1. INTRODUCTION.

Throughout this paper, $R$ will be an associative ring, $Z(R)$ the center of $R$, $N$ the set of all nilpotent elements of $R, N^{\prime}$ the set of all zero divisors of $R$, and $C(R)$ the commutator ideal of $R$. For any pair of elements $x, y$ in $R$, we set as usual $[x, y]=x y-y x$.

Recently, generalizing some results from Bell [1] Quadri and Khan [2,3]) proved that if $R$ is a ring satisfying the polynomial identity $\left[x y-y^{m} x^{m}, x\right]=0$ ( $m>1, n>1)$, then $R$ is commutative. In [4], Psomopoulos has shown that an s-unital ring $R$ in which the polynomial identity $\left[x^{n} y-y^{m} x, x\right]=0(m>1, n>0)$ holds, must be commutative.

In this paper, motivated by the above polynomial identities, we intend to prove results on commutativity of a ring $R$ with unity satisfying the fullowing property:

(i) "there exist positive integers $m>1$ and $n>1$ such that $\left[x^{n} y-y^{m} x^{n}, x\right]=0$ for al1 $x, y$ in $R^{\prime \prime}$.

Our property (i) can be regarded as an amalgam of those consider: 1 by the above authors. 


\section{PRELIMINARIES.}

In preparation for the proof of our results, we first state the following wellknown results.

LEMMA 2.1. (Psomopoulos [4]). Let $x, y \in R$. If $[x, y], x=0$, the for any positive integer $k,\left[x^{k}, y\right]=k x^{k-1}[x, y]$.

LEMMA 2.2. (Nicholson and Yaqub [5]). Let $R$ be a ring with unity 1 . Suppose that for some positive integer $k, x^{k} y=0=(x+1)^{k} y$ for all $x, y$ in $R$. The $y=0$.

LEMMA 2.3. (Bell [6]). Let $\mathrm{f}$ be a polynomial identity in a finite number of noncommuting indeterminates with integral coefficients. Then the following are equivalent:

(i) For any ring $R$ satisfying $f=0, C(R)$ is a nil ideal.

(ii) For every prime $p,(G F(p))_{2}$ fails to satisfy $f=0$.

LEMMA 2.3. (Tong [7]). Let $R$ be a ring with unity 1 . Let $I_{0}^{r}(x)=x^{r}$. If $k>1$, let $I_{k}^{r}(x)=I_{k-1}^{r}(x+1)-I_{k-1}^{r}(x)$. Then $I_{r-1}^{r}(x)=1 / 2(r-1) r$ ! $+r ! x ; I_{r}^{r}(x)=r$ ! , and $I_{j}^{r}(x)=0$ for $j>r$.

\section{RESULTS.}

Throughout the rest of the paper, $R$ stands for a ring with unity 1 , and satisfies the property (1). Let us first note that for any $x$, $y$ in $R$, the property (i) can also be expressed as:

$$
x^{n}[x, y]=\left[x, y^{m}\right] x^{n} \text {. }
$$

Then for any positive integer $t$, we obtain

$$
\begin{aligned}
& x^{t n}[x, y]=x^{(t-1) n}\left[x, y^{m}\right] x^{n}=x^{(t-2) n} \\
& {\left[x, y^{m^{2}}\right] x^{2 n}=x^{(t-3) n}\left[x, y^{m^{3}}\right] x^{3 n}=}
\end{aligned}
$$

By repeating the above process and using ( 3.1$)$, we get

$$
x^{t n}[x, y]=\left[x, y^{m^{t}}\right] x^{t n} \text {. }
$$

We also need the following two results for the proof of our main theorem.

LEMMA 3.1. Let $R$ be a ring with unity which satisfies the property (P). Then $N$ $Z(R)$.

PROOF. Let $u \in N$. Then by (3.2) for any $x \in R$ and a positive integer $t$, we have

$$
x^{t n}[x, u]=\left[x, u^{m}\right] x^{t n}
$$

But we have $u$ as a nilpotent element, then $u^{m^{t}}=0$, for sufficiently large $t$. Therefore, $x^{t n}[x, u]=0$ for all $x$ in $R$. Then we have $(x+1)^{t n}[x, u]=0$ for all $x$ in R. By Lemma 2.2, this implies that $[x, 11]=0$, which forces $N Z(R)$.

LEMMA 3.2. Let $R$ be a ring with unity which satisfies the property (i). Then $C(R) \quad Z(R)$. 
PROOF. Replacing $x$ by $(x+1)$ in (3.1) and inultiplying both sides by $x^{n}$ on the right and again using (3.1), we get

$$
(x+1)^{n}[x, y]^{n}=x^{n}[x, y](x+1)^{n} \text {, for } x, y \in R .
$$

Define

$$
E_{11}=\left(\begin{array}{ll}
1 & 0 \\
0 & 0
\end{array}\right), \quad E_{12}=\left(\begin{array}{ll}
0 & 1 \\
0 & 0
\end{array}\right) \text {, and } E_{21}=\left(\begin{array}{ll}
0 & 0 \\
1 & 0
\end{array}\right) \text {. }
$$

Let $x=E_{11}+E_{12}+E_{12}$ and $y=E_{11}$. Then $x$ and $y$ fail to satisfy (3.3) in $(G F(p))_{2}$, for a prime p. So by Lemma $2.3, C(R)$ is a nil ideal, and hence by Lemma 3.1 , $C(R) Z(R)$. This ends the proof.

In view of Lemma 3.2, it is guaranteed that the condition of Lemma 2.1 holds for each pair of elements $x, y$ in a ring $R$ with unity which satisfies the property (i).

THEOREM 3.1. Let $R$ be a ring with unity 1 satisfying property

(i) Then $R$ is commutative.

PROOF. We are given that $R$ is a ring with unity. So $R$ is isomorphic to a subdirect sum of subdirectly irreducible rings $R_{i}$ ( $i \varepsilon I$ ), each of which as a homomorphic image of $R$, satisfing the property (i). Thus we may assume that $R$ is a subdirectly irreducible ring satisfying (i).

Let $S$ be the intersection of all non-zero ideals of $R_{1}$. Then clearly $S \neq(0)$.

Now, if $n=1$ in the polynomial identity (3.1), we obtain $\left[x, y-y^{m}\right]=(x+1)[x, y]$ $-\left[x, y^{m}\right](x+1)-x[x, y]-\left[x, y^{m}\right] x=0$ for all $x, y \varepsilon R$. Thus $R$ is commutative by Herstein [8, Theorem 18].

Henceforth we assume $n>1$. Consider the positive integer $k=p^{m}-p$, where $p$ is a positive integer greater than 1 . Then by (3.1) we get

$$
\begin{aligned}
k x^{n}[x, y] & =p^{m} x^{n}[x, y]-p x^{n}[x, y] \\
& =p^{m}\left[x, y^{m}\right] x^{n}-p x^{n}[x, y] \\
& =\left[x,(p y)^{m}\right] x^{n}-x^{n}[x,(p y)] \\
& =x^{n}[x,(p y)]-x^{n}[x,(p y)]
\end{aligned}
$$

Thus $k x^{n}[x, y)=0$, whcih on replacing $x$ by $(x+1)$ yields $k[x, y]=0$. Now combining Lemma 3.2 with Lemma 2.1 , we have $\left[x^{k}, y\right]=k x^{k-1}[x, y]=0$. Therefore, it follows that

$$
x^{k} \varepsilon Z(R) \text { for all } x \text { in } R \text {. }
$$

Now, replacing $y$ by $y^{m}$ in $(3.1)$ we get

$$
x^{n}\left[x, y^{m}\right]=\left[x,\left(y^{m}\right)^{m}\right] x^{n} .
$$

Then by Lemma 3.2 , we have

$$
x^{n}\left[x, y^{m}\right]=\left[x, y^{m}\right] x^{n} .
$$

But

$$
\left[x, y^{m}\right]=m y^{m-1}[x, y]
$$


So using (3.6) and (3.7) we obtain

$$
x^{n}\left[x, y^{m}\right]=m y^{m-1}\left[x ; y^{m}\right] x^{n},
$$

and

$$
\begin{aligned}
{\left[x,\left(y^{m}\right)^{m}\right] x^{n} } & =m\left(y^{m}\right)^{m-1}\left[x, y^{m}\right] x^{n} \\
& =m y^{m-1} y^{(m-1)^{2}}\left[x, y^{m}\right] x^{n} .
\end{aligned}
$$

Thus (3.5) gives

$$
m y^{m-1}\left(1-y(m-1)^{2}\right) \quad\left[x, y^{m}\right] \quad x^{n}=0 .
$$

Let us replace $x$ by $(x+1)$ in $(3.8)$. Then we get $m y^{m-1}\left(1-y(m-1)^{2}\right)\left[x, y^{m}\right](x+1)^{n}=0$. So by Lemma $2.2, \mathrm{~m} \mathrm{y}^{\mathrm{m}-1}\left(1-\mathrm{y}^{(\mathrm{m}-1)^{2}}\right)\left[\mathrm{x}, \mathrm{y}^{\mathrm{m}}\right]=0$. Therefore, by Lemma 2.3 of Quadri and Khan [3], we have

$$
m y^{m-1}\left(1-y^{k(m-1)^{2}}\right)\left[x, y^{m}\right]=0 \text {. }
$$

Now, let $u \in N^{\prime}$. Then by (3.4), $u^{k(m-1)^{2}} \varepsilon N^{\prime} Z(R)$, and $s u^{k(m-1)^{2}}=(0)$. Hence using (3.9) we obtain

$$
m u^{m-1}\left[x, u^{m}\right]\left(1-u^{k(m-1)^{2}}\right)=0 \text {. }
$$

If $m u^{m-1}\left[x, u^{m}\right] \neq 0$, then $\left(1-u^{k(m-1)^{2}}\right) \in N^{\prime}$ and so $S=S\left(1-u^{k(m-1)^{2}}\right)=(0)$, which gives a contradiction as $S \neq(0)$. Therefore

$$
m u^{m-1}\left[x, u^{m}\right]=0 \text {. }
$$

Now, from (3.1) we have

$$
\begin{aligned}
x^{2 n}[x, u] & =\left[x, u^{m^{2}}\right] x^{2 n} \\
& =m u^{m(m-1)}\left[x, u^{m}\right] x^{2 n} \\
& =m u^{m-1} u^{(m-1)^{2}}\left[x, u^{m}\right] x^{2 n} \\
& =m u^{m-1}\left[x, u^{m}\right] u^{(m-1)^{2}} x^{2 n} .
\end{aligned}
$$

This implies that $x^{2 n}[x, u]=0$. Hence by Lemma 2.2 , we obtain $[x, u]=0$, that is

$u \in Z(R)$. Therefore, $N^{\prime} Z(R)$.

Clearly, for any $x \in R, x^{k}$ and $x^{k m} \varepsilon Z(R)$. Then by (3.1) for any $y \varepsilon R$, we have the identity

$$
\begin{aligned}
\left(x^{k}-x^{k m}\right) x^{n}[x, y] & =x^{k}\left(x^{n}[x, y]\right)-x^{k m}\left(x^{n}[x, y]\right) \\
& =x^{n}\left(x^{k}[x, y]\right)-\left(x^{k m}[x, y]\right) x^{n} \\
& =x^{n}\left[x, x^{k} y\right]-\left[x,\left(x^{k} y\right)^{m}\right] x^{n} \\
& =x^{n}\left[x, x^{k} y\right]-x^{n}\left[x, x^{k} y\right]
\end{aligned}
$$

Therefore,

$$
\begin{aligned}
& \left(x^{k}-x^{k m}\right) x^{n}[x, y]=0 \text {, and } \\
& \left(x-x^{t}\right) x^{s}[x, y]=0,
\end{aligned}
$$

where $t=k m-k+1$, and $s=n+k-1$. 
Now, if $x^{s}[x, y]=0$, then Lemma 2.2 yields $[x, y]=0$. But $x^{s}[x, y] \neq 0$ gives $x-x^{t} \in N^{\prime} \quad Z(R)$.

Therefore, $\left[x-x^{t}, y\right]=0$, for all $x, y$ in $R$, which implies that $[x, y]=0$, by Theorem 18 of [9]. Thus in evey case $[x, y]=0$.

This proves that $R$ is commutative.

THEOREM 3.2. Let $R$ be an s-unital ring satisfying the property ( 1 ). Then $R$ is commutative.

PROOF. This follows from Proposition 1 of Hirano, Kobayashi and Tominaga [9] since $R$ with unity satisfying (i) is commutative by Theorem 3.1. Finally, we present a short and easy proof of Theorem 3.1, but under an extra condition on the commutators in the ring $R$. We use an iteration technique as given in Tong [7].

THEOREM 3.3. Let $R$ be a ring with unity satisfying the property (i). If every commutator in $R$ is $m$ ! - torsion free, then $R$ must be commutative.

PROOF. The ring $R$ satisfies the identity

$$
\left.x^{n}[x, y]=\left[x, y^{m}\right] x^{n}, n>1, m>1\right) .
$$

We shall apply the iteration on $y^{m}$. As in $[7]$, let $I_{j}(y)=I_{j}^{m}(y)$ for $j=0,1,2, \ldots$. Then the above identity can be rewritten as

$$
x^{n}[x, y]=\left[x, I_{0}(y)\right] x^{n}
$$

Replacing $y$ by $(y+1)$ in $(3.11)$, we obtain

$$
x^{n}[x, y]=\left[x, I_{0}(y+1)\right] x^{n} .
$$

Now, using Lemma 2.4, we get

$$
x^{n}[x, y]=\left[x, I_{0}(y)+I_{1}(y)\right] x^{n} .
$$

Equations (3.11) and (3.12) when combined, give

$$
0=\left[x, I_{1}(y)\right] x^{n} \text {. }
$$

Again, let $y=y+1$ in (3.13). Then using Lemma 2.4 we have

$$
0=\left[x, I_{2}(y)\right] x^{n} \text {. }
$$

Repeating the above process $(m-1)$ times, we reach the identity

$$
0=\left[x, I_{m-1}(y)\right] x^{n} \text {. }
$$

With an application of Lemma 2.4 , we end up with

$$
\mathrm{m} ![\mathrm{x}, \mathrm{y}] \mathrm{x}^{\mathrm{n}}=0 \text {. }
$$

Now replacing $x$ by $(x+1)$ in the above identity, and making ue of Lemma 2.2 , we have

$$
m ![x, y]=0 \text {, for all } x, y \in R \text {. }
$$

By every commutator in $R$ is $m$ !-torsion free, so $[x, y]=0$ for all $x$ and $y$ in $R$. Therefore, $R$ is commutative. This completes the proof. 


\section{REFERENCES}

1. BELL, H.E., Some commutativity results for rings with two variable constraints, Proc. Amer. Math. Soc. 53 (1975), 280-285.

2. QUADRI, M.A. and KHAN, M.A., A commutativity theorem for associative rings, Math. Japon 33(2) (1988), 275-279.

3. QUADRI, M.A. and KHAN, M.A., A commutativity theorem for left s-unital rings, Bu11. Inst. Math. Acad. Sinica, 15(3) (1987), 323-327.

4. PSOMOPOULOS, E., A commutativity theorem for rings, Math. Japon 29 (1984), 371373.

5. NICHOLSON, W.K. and YAQUB, A., A commutativity theorem for rings and groups, Canad. Math. Bul1. 22 (1979), 419-423.

6. BELL, H.E., On some commutativity theorems of Herstein, Arch. Math. 24 (1973), 3438.

7. TONG, J., On the commutativity of a ring with identity, Canad. Math. Bull. 27(4) (1984), 456-460.

8. HERSTEIN, I.N., A generalization of a theorem of Jacobson, Amer. J. Math. 73 $(1951), 756-762$.

9. HIRANO, Y., KOBAYASHI, Y. and TOMINAGA, H., Some polynomial identities and commutativity of s-unital rings, Math. J. Okayama Univ. 24 (1982), 7-13. 


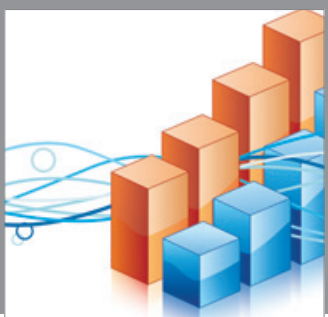

Advances in

Operations Research

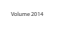

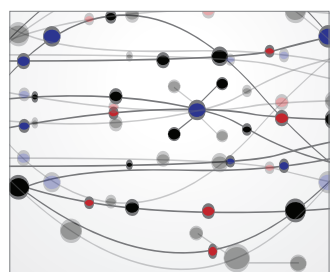

\section{The Scientific} World Journal
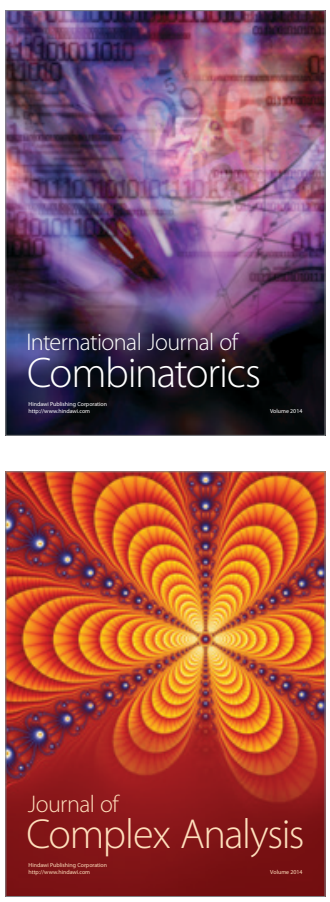

International Journal of

Mathematics and

Mathematical

Sciences
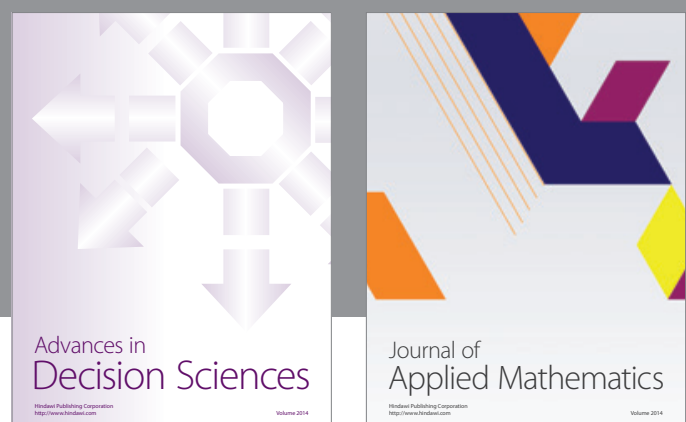

Journal of

Applied Mathematics
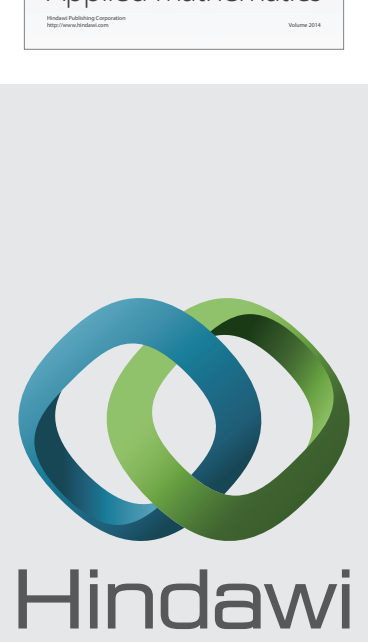

Submit your manuscripts at http://www.hindawi.com
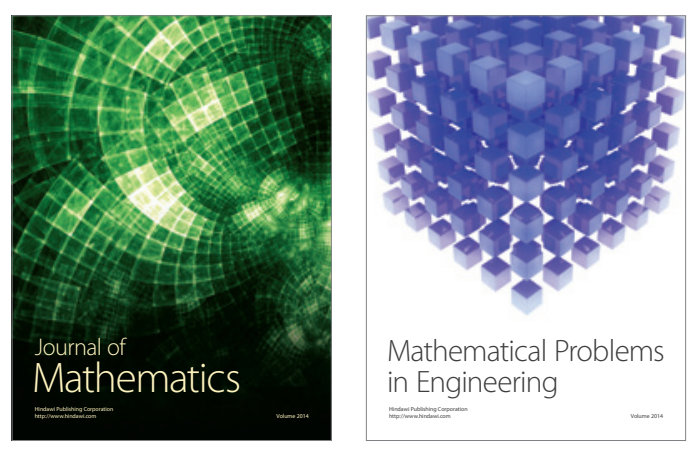

Mathematical Problems in Engineering
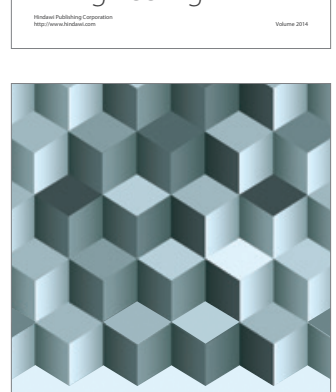

Journal of

Function Spaces
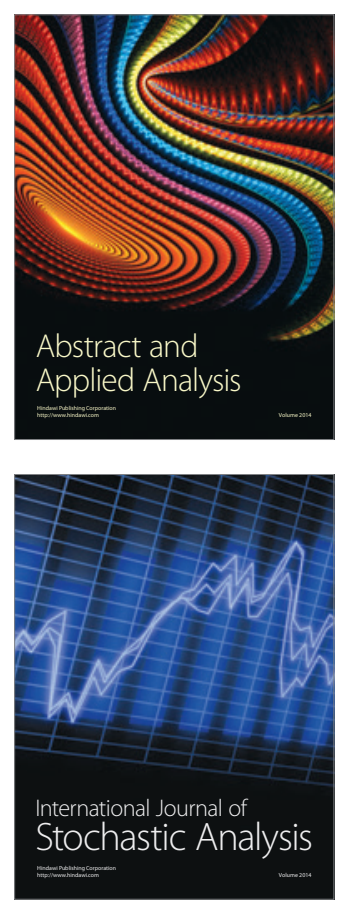

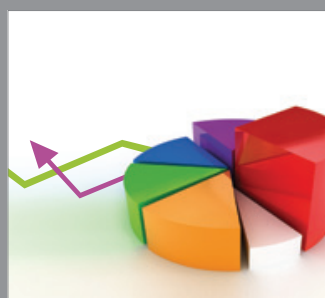

ournal of

Probability and Statistics

Promensencen
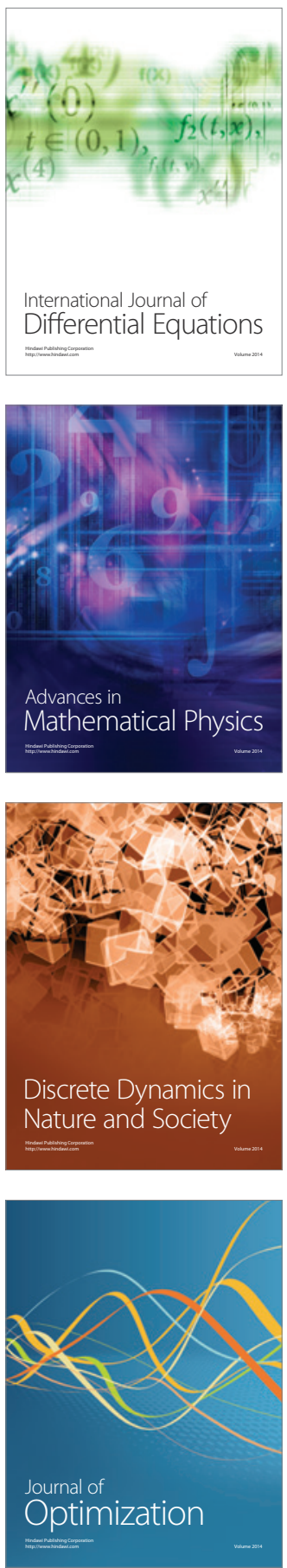